\title{
Energy Storage Systems to prevent distribution transformers overload with high NZEB penetration
}

\author{
Renato Veríssimo \\ $\mathrm{DEE} / \mathrm{FCT} / \mathrm{UNL}$ \\ Lisbon, Portugal \\ r.verissimo@campus.fct.unl.pt
}

\author{
Rui Amaral Lopes \\ $\mathrm{DEE} / \mathrm{FCT} / \mathrm{UNL}$ \\ CTS/UNINOVA \\ Lisbon, Portugal \\ rm.lopes@fct.unl.pt
}

\author{
João F. Martins \\ $\mathrm{DEE} / \mathrm{FCT} / \mathrm{UNL}$ \\ CTS/UNINOVA \\ Lisbon, Portugal \\ jf.martins@fct.unl.pt
}

\begin{abstract}
This paper studies and analyses the impacts introduced on Distribution Transformer (DT) overload due to the integration of nearly Zero-Energy Buildings (nZEBs) into existing distribution Low Voltage Grids (LVG). The negative impacts on the DT are studied and explained. For high nZEB integration levels, this study reveals that the subsequent reverse power flows can achieve higher values, especially around noon, than the DT rated (and overload limit) power thus accelerating its aging. A power electronics based Energy Storage System (ESS) is considered to mitigate these negative effects. The paper also analysis the mitigation of the negative DTs impact by considering this ESS solution. The established control laws prevent the transformer to be exposed to excessive reverse power flow levels.
\end{abstract}

Keywords- energy storage system, NZEB, distribution transformer, low voltage grid

\section{INTRODUCTION}

One of the key targets of the European Union (EU) is to reduce by $88-91 \%$ its amount of $\mathrm{CO} 2$-equivalent emissions by 2050 , considering as background its 1990 values [1]. Buildings account for $40 \%$ of energy consumption and $36 \%$ of CO2-equivalent emissions in the EU [2][3]. In order to minimize the ecological footprint and ensure a sustainable energy sufficiency this problem requires deep and major refurbishment and improvements of the building stock [4]. A key issue to solve this problem relies on the Nearly Zero Energy Buildings concept (NZEB). There are several NZEB definitions, however in this paper the following will be considered: "nearly zero-energy building means a building that has a very high energy performance. The nearly zero or very low amount of energy required should be covered to a very significant extent by energy from renewable sources, including energy from renewable sources produced on-site or nearby" [5]. The EU Energy Performance in Buildings Directive (EPBD) mandates that all new buildings, built from 2021 onwards, should be at least NZEB [5]. Furthermore, this same directive endorses EU member states to set specific targets in order to encourage the conversion of regular buildings into NZEB whenever those are subjected to some kind of renovation. It is expected that the adoption of this recommendation will enlarge the NZEB adoption in several countries, particularly on those where large numbers of old buildings need rehabilitation interventions. In the EU, the majority of the buildings are more then 30 years old and about $35 \%$ are more than 60 years old.
As an example, in Portugal about $50 \%$ of the building stock is more than 30 years old [6].

On a simpler formulation one can state that a NZEB is a building presenting a nearly zero annual balance between energy demand and on-site energy production. Being this computation taken on an annual basis the real time computation is obviously non zero. Considering a household with the typical load diagram presented in Fig. 1, located in the centre of Portugal, a $1.8 \mathrm{~kW}$ solar Photovoltaic (PV) system is needed in order to consider this household as NZEB. In Fig. 1, the black line denotes the home consumption and the grey line denotes the on-site PV power production.

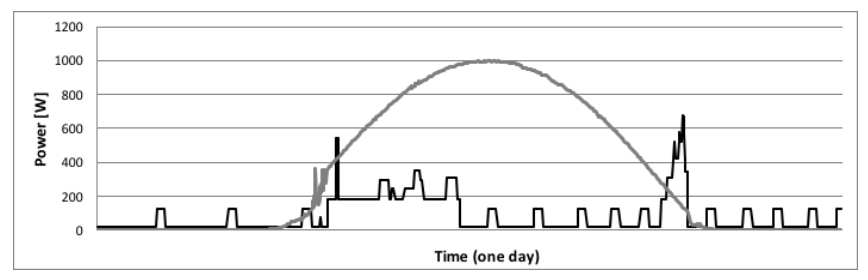

Fig. 1. Household typical load diagram.

With this PV system installed, the NZEB will present a seasonal grid interaction behaviour. The overall import/export energy computation is nearly zero, but there is a clear mismatch producing a net export in the day time and a net import in the rest of the day, as can be seen in Fig. 2 where the net balance is presented.

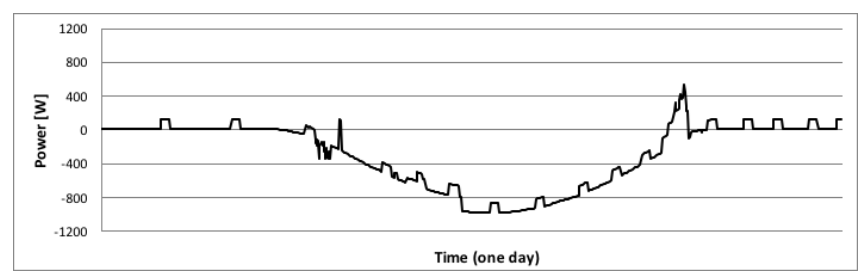

Fig. 2. Monthly load and generation.

As can be seen in Fig. 2, the household reverse energy flow can achieve higher values than the direct flow. It is important to note that these households are integrated on a low-voltage grid (LVG) where Distribution Transformers (DT) are usually designed in order to meet the maximum load on the grid (with some security factor). 
With daily net energy unbalance the distribution transformer must also be able to handle this reverse flow upstream from the LVG end-users. By end-users one considers the several NZEB spread around the LVG. The introduction of NZEB (considering the adaptation of the EPBD directive) implies the introduction of new distributed generation into already existing LVGs, whose DTs were originally designed to deal only with unidirectional power flows. This NZEB introduction will create impacts which depend on the amount of distributed generation and also on the used power electronics interface [7]. Furthermore the DTs' aging can be highly increased [8][9].

This paper studies the effects of increasing NZEB penetration on LVG, particularly on the DTs overload. It also proposes an energy storage based solution in order to mitigate the negative impacts that increasing NZEB penetration have on the DT. The remaining of this paper is structured as follows: Section 2 addresses LVGs with high penetration of NZEBs, Section 3 presents the considered case study, comprising the description of the Energy Storage based solution, and Section 5 presents conclusions and future work.

\section{LVGS WITH HIGH NZEB PENETRATION}

Low voltage distribution grids are historically design in such a way that the power flow is considered to occur from the medium voltage grid towards the low voltage grid and thus feeding end user consumers [10]. The recent integration of distributed generation systems (particularly PV systems) in the low voltage grids has caused serious problems on the behaviour of the traditional distribution feeders. Being a good solution from the energy sustainability point of view, low voltage PV systems can also impose several negative impacts on the electrical system, particularly if their penetration level is high. These PV systems can go from small (less than $10 \mathrm{~kW})$ to intermediate $(10-500 \mathrm{~kW})$, which are both usually installed at the distribution level (low voltage). Large PV systems (higher than $500 \mathrm{~kW}$ ) are usually installed at medium level.

As mentioned in the previous section, when converting a regular building into the NZEB, the building is set to act as a prosumer. The usual addition of PV systems makes it to act as consumer/producer in different times of year, month or day. The analysis of the NZEB impact on the LVG is more complicated than the analysis of the impact of distributed PV systems, because in the NZEB case one has to consider, not only the solar radiation, but also the occupancy and load profiles. This makes this analysis a stochastic one because occupancy is not as deterministic as solar radiation. However, taking into account the net balance, when exporting to the grid a NZEB can cause the same negative impacts as a non-integrated PV production system.

Many authors have studied the possible negative impacts of distributed generation penetration. With a high distributed generation penetration degree one can observe reverse power flow in the LVG, overloading distribution feeders and increasing power losses [11]. This reverse power flow can lead to an overvoltage effect along the distribution feeders
[12]. Distributed generation systems (and also NZEBs) can increase load match thus reducing energy distribution losses. However, this is only true if reverse power flow does not occur. Also the existence of more that one power injection node will cause controllability problems on the LVG. Singlephase distributed generation systems surely will cause phase unbalance on the three-phase LVG. Although some advanced PV inverters automatically balance the load they are only suitable for three-phase PV systems [13], which is not the case of most households. PV power electronic converters, although getting better every time, often cause harmonics' problems on the LVG. Since most of the small and intermediate distributed generation systems do not perform reactive power injection (although some of them are able to most of the contracts do not foreseen that in LVG) the power factor lowers in the LVG affecting the DTs efficiency [14].

A major negative impact of distributed generation penetration, and in particular of the foreseen increasing number of NZEB buildings as LVG loads, is the excessive aging of Distribution Transformers due the existence of reverse power flow higher than their rated power. As mentioned Distribution Transformers are usually design in order to meet the maximum load on the grid not considering the existence of reverse power flow. However, this reverse power flow can be occasionally higher that the DTs' power rate. This negative impact can become dangerously severe if one considers that all buildings in the LVG feeder are sustainable being converted into NZEB. Than, it is not only the impact of one building (with its reverse power flow) but one has to consider this negative impact emerging from all nodes on the LVG.

Furthermore, when several buildings in the same LVG are converted into NZEB one has to consider the effects of load and generation coincidence. If the loads of an aggregation of NZEBs can be considered stochastic, thus smoothing the overall load, that can not be considered for their PV generation, because this has a simultaneous occurrence effect [15].

As detailed in the following sections this negative impact on the Distribution Transformer can be mitigated with a centralized energy storage system based solution.

\section{CASE STUDY}

The case study presented in this paper focus a LVG located in Lisbon, Portugal, supplying 33 buildings and aims to: i) show the impact introduced by the NZEBs' on-site generation on the transformer's load; and ii) study the operation of a power electronics based Energy Storage System (ESS) to mitigate transformer's overload when a relatively high level of NZEBs integrate the LVG. To address the first objective, different scenarios are considered, being the difference between scenarios related with the number of original buildings converted to NZEB using onsite PV generation (i.e. one additional building converted to NZEB per scenario). The baseline scenario refers to a neighborhood composed only by regular buildings that do not generate energy on-site. To address the second objective, an extra scenario is used, where all the 33 buildings are 
NZEBs and an ESS is integrated to mitigate transformer's overload. This ESS is located at the transformer's output and, from the transformer's point of view, is seen as a consumption device when charging and as a virtual generation device when discharging. The main objective is to charge the ESS during periods with reverse power flow values higher than a predefined limit and to discharge it when demand is higher than generation. Another possibility was to consider distinct ESS along the LVG, each one of them integrated into each NZEB, considering energy router based solutions [16] or multiport interface based solutions [17]. However, this is not the best solution when small scale microgrids (as this 33 use case LVG) are considered. For this study a standard AC/DC bidirectional power electronics converter was considered as gateway between the transformer's output and the energy storage system, denoted as ESS in Fig. 3.

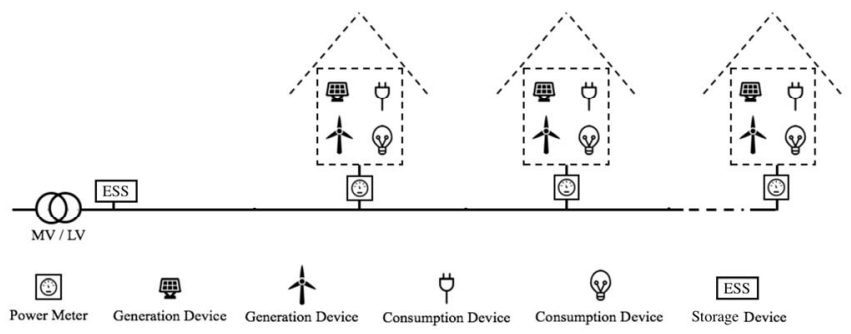

Fig. 3. Use case NZEB LVG.

For all the considered scenarios, 1-year simulations are carried out using 15-min resolution data and one representative day per month. Additionally, each building's load diagram refers to the difference between building's demand and on-site PV generation profiles (when converted to NZEB). In this study, Richardson's model is used to generate the demand profiles of each 24-h load cycle [18]. To collect each demand profile, appliances were randomly allocated to the dwellings and an occupancy model was considered for each building [19]. Due to this occupancy dependence, the probability that some devices have to operate depends on the number of occupants of the respective building. Therefore, the electricity demand of a specific building is also dependent on its number of occupants, as well as if it is a weekday or a weekend. It was considered that the simulation took place during week days and that the number of residents in each building vary from 1 to 5 . To set the number of occupants for each building, an uniform distribution was used. Regarding the on-site PV generation profile, the tool provided in [20] was used. In order to convert the global irradiance on a fixed plane with optimized inclination and azimuth to active power, considering also the average daytime temperature profile, a nominal operating cell temperature (NOCT) of $45^{\circ} \mathrm{C}$ and a temperature coefficient of the maximum output power (at STC conditions) of $-0.45 \% /{ }^{\circ} \mathrm{C}$ were considered for all buildings. The ESS power electronics converter efficiency value was set to $97 \%$ and it was also assumed installation losses of $15 \%$. In each building converted to NZEB, the installed peak power was linearly scaled in order to occur a net-zero energy balance over the analysed year (i.e. the annual energy generated on-site equals the building's annual energy demand). The main characteristics of each building are presented in TABLE I., focusing their position on the LVG, the considered number of occupants, the annual energy demand and the installed peak power in order to convert each building to NZEB.

\section{RESULTS AND ANALYSIS}

This section starts by addressing the results related with the neighbourhood composed by only regular buildings, which do not generate energy on-site (i.e. the baseline scenario). Then, results associated with the conversion of regular buildings to NZEB are presented and analysed. Lastly, the impact introduced by the ESS is assessed, assuming that the proposed system is integrated into the LVG (at transformer output) when all buildings are NZEBs in order to mitigate transformer's increased overload.

TABLE I. BUILDING's CHARACTERISTICS

\begin{tabular}{|c|c|c|c|}
\hline \multirow{2}{*}{$\begin{array}{l}\text { LVG } \\
\text { node }\end{array}$} & \multicolumn{3}{|c|}{ Building characteristics } \\
\hline & Occupants & $\begin{array}{c}\text { Annual demand } \\
{[\mathrm{kWh}]}\end{array}$ & $\begin{array}{c}\text { PV peak power } \\
{[\mathrm{kW}]}\end{array}$ \\
\hline 1 & 1 & 2040.377 & 1.31 \\
\hline 2 & 1 & 2384.762 & 1.54 \\
\hline 3 & 1 & 2643.145 & 1.70 \\
\hline 4 & 2 & 3235.576 & 2.07 \\
\hline 5 & 3 & 3809.136 & 2.44 \\
\hline 6 & 3 & 3569.799 & 2.29 \\
\hline 7 & 2 & 3780.279 & 2.44 \\
\hline 8 & 5 & 3484.524 & 2.24 \\
\hline 9 & 3 & 3351.276 & 2.15 \\
\hline 10 & 1 & 2528.864 & 1.62 \\
\hline 11 & 2 & 2777.984 & 1.79 \\
\hline 12 & 2 & 3370.881 & 2.16 \\
\hline 13 & 2 & 3177.001 & 2.04 \\
\hline 14 & 1 & 2610.836 & 1.69 \\
\hline 15 & 3 & 3378.39 & 2.17 \\
\hline 16 & 4 & 4358.604 & 2.80 \\
\hline 17 & 5 & 4122.102 & 2.65 \\
\hline 18 & 1 & 3170.14 & 2.03 \\
\hline 19 & 3 & 2792.64 & 1.78 \\
\hline 20 & 5 & 4504.445 & 2.89 \\
\hline 21 & 4 & 4163.692 & 2.67 \\
\hline 22 & 3 & 3885.875 & 2.50 \\
\hline 23 & 4 & 3456.558 & 2.21 \\
\hline 24 & 4 & 4291.5 & 2.75 \\
\hline 25 & 5 & 4550.636 & 2.91 \\
\hline 26 & 2 & 2862.831 & 1.84 \\
\hline 27 & 4 & 3847.933 & 2.48 \\
\hline 28 & 5 & 4308.402 & 2.76 \\
\hline 29 & 4 & 3537.339 & 2.27 \\
\hline 30 & 3 & 3355.519 & 2.16 \\
\hline 31 & 5 & 4208.333 & 2.72 \\
\hline 32 & 4 & 3479.817 & 2.23 \\
\hline 33 & 5 & 4509.146 & 2.88 \\
\hline
\end{tabular}




\section{A. Baseline Scenario}

The load profile of the studied LVG, measured at distribution transformer's output, typically follows a $24 \mathrm{~h}$ duration cycle. As presented in Fig. 4, which shows the load profile at transformer's output for each representative day of each month, the original load cycles are characterized by a reduced power consumption value during night followed by a morning peak at breakfast time. During the day, the load profiles show a relatively levelled power consumption, until mid-afternoon, when it rises towards the evening peak. After this second peak, LVG's electricity demand falls again reaching the night-time lower values.

According to IEC 60076-7 standard [21], normal operation of a distribution transformer is compatible with loads up to $50 \%$ above the respective rated load. Therefore, considering the results presented in Fig. 4, a $24.9 \mathrm{~kW}$ transformer would be needed to guarantee a normal operation during the considered year, since the registered yearly peak load was $37.3 \mathrm{~kW}$. In terms of transformer aging, such overloading is normally allowed in distribution grids because operation periods above the rated load are compensated by other periods with load values lower than the rated one [21]. This is clearly illustrated in Fig. 4, where, for instance, night time load values are much lower than the ones registered during evening.

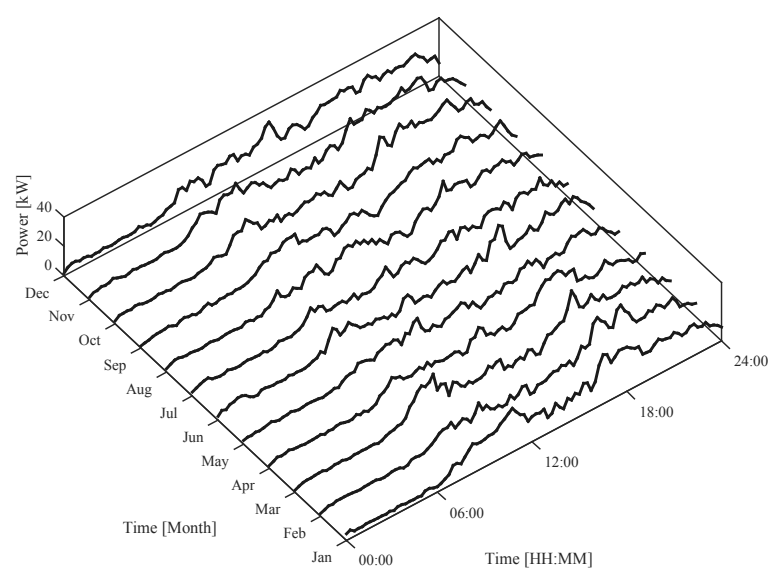

Fig. 4. LVG's load at distribution transformer's output for the baseline scenario.

\section{B. Increasing NZEB Penetration}

The integration of PV-based energy conversion systems, in each building converted to NZEB, considerably impacts the presented $24 \mathrm{~h}$ duration load cycles. These impacts are registered during daytime, when the solar resource is available, and strongly depend on the number of buildings converted to NZEB. To illustrate the impacts introduced on LVG's load, Fig. 5 presents the yearly peak load at transformer's output. This figure shows that the introduced impacts can be both positive and negative, depending on the number of buildings converted to NZEBs. For a reduced number of converted buildings, the yearly peak load decreases, reaching a minimum of $35.5 \mathrm{~kW}$ when 13 NZEBs integrate the LVG. This is due to the magnitude reduction of the load profiles during daytime, even in the presence of reverse power flow. From 13 to 30 NZEBs, no further reduction is achieved since the resulting peak load occurs during the evening, despite the significant load reduction around noon. When more than 30 buildings are converted to NZEB, yearly peak load values increase until reaching a maximum of $40.7 \mathrm{~kW}$, when the 33 buildings achieve the NZEB condition. This yearly peak load increasing is due to the coincident on-site generation of the NZEBs, which results on reverse power flows with magnitudes higher than the evening related demand peak loads.

The combined household reverse energy flow achieves higher values than the direct flow, at transformer's output. This impact can change day-by-day depending on the PV generation and on the stochastic load behaviour (highly depended on the external temperature and user behaviour). This means that one can achieve lower of much higher negative impacts (comparing with the ones presented here) depending on this stochastic behaviour.

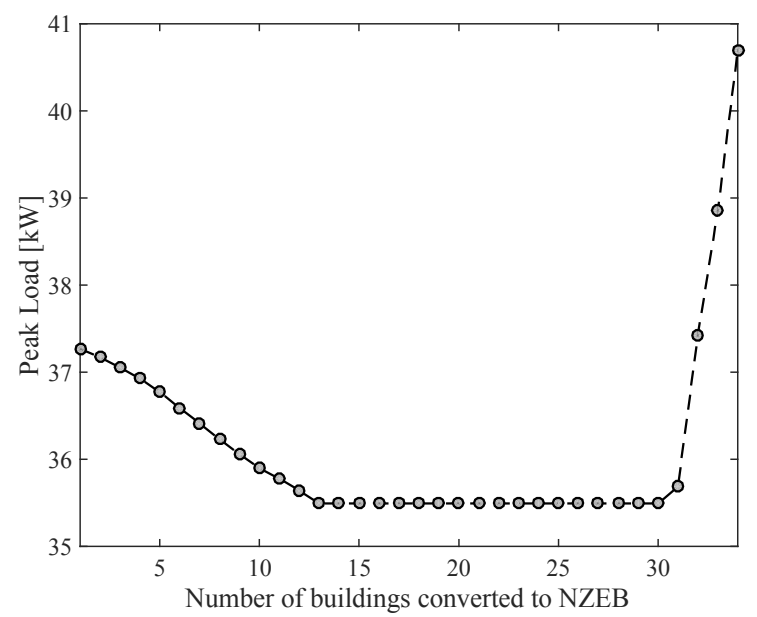

Fig. 5. LVG's yearly peak load.

Fig. 6 presents the LVG's load at transformer's output during the analyzed year for the most problematic scenario in terms of yearly peak load (i.e. when all buildings are in a NZEB condition). The impacts introduced by the coincident PV generation are evident. In this case, transformer's load achieves values higher than the maximum obtained for the baseline during July, August and September. Therefore, comparing to the baseline scenario, a $24.9 \mathrm{~kW}$ transformer would be exposed to load values beyond the limit for a normal operation, which could result on transformer permanent failure or excessive aging due to thermal stress [22].

\section{Energy Storage based solution results}

This section presents the obtained results when the ESS installed at the transformer's output is used to mitigate the negative impacts introduced on the distribution transformer's load by the conversion of regular buildings to NZEBs. The worst case scenario, when all the 33 buildings are converted, 
is considered. The $24.9 \mathrm{~kW}$ transformer, associated to a normal operation for the baseline scenario, is assumed to integrate this LVG. The ESS control algorithm is established in order to prevent the overload of the distribution transformer. In this way the power electronics converter should obey the following rules:

- In presence of excess reverse power flow, the power electronics convert should re-direct this excess into the energy storage system;

- No charge or discharge will be performed when the exported power is within distribution transformer operating limits;

- The energy storage system will inject power whenever energy is being imported from the grid or whenever the DSO asks for a consumption reduction (possible ancillary services provided by the microgrid).

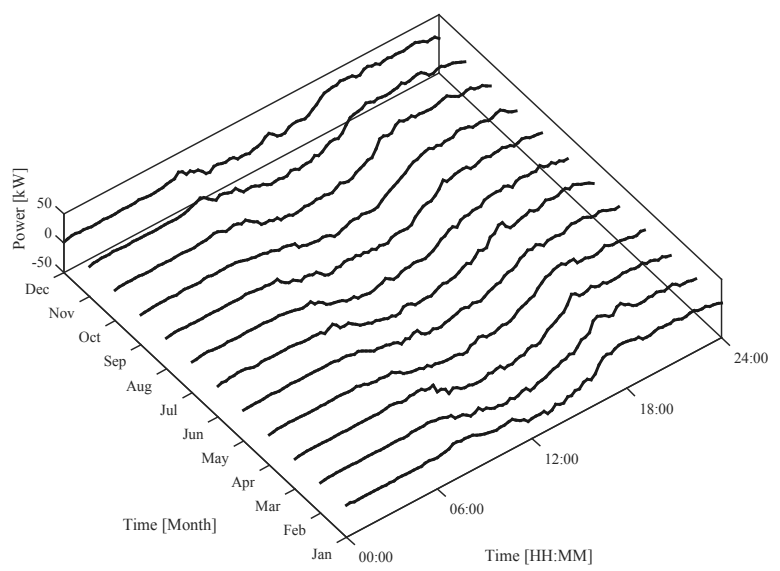

Fig. 6. LVG's load measured at distribution transformer's output when the 33 buildings are converted to NZEBs.

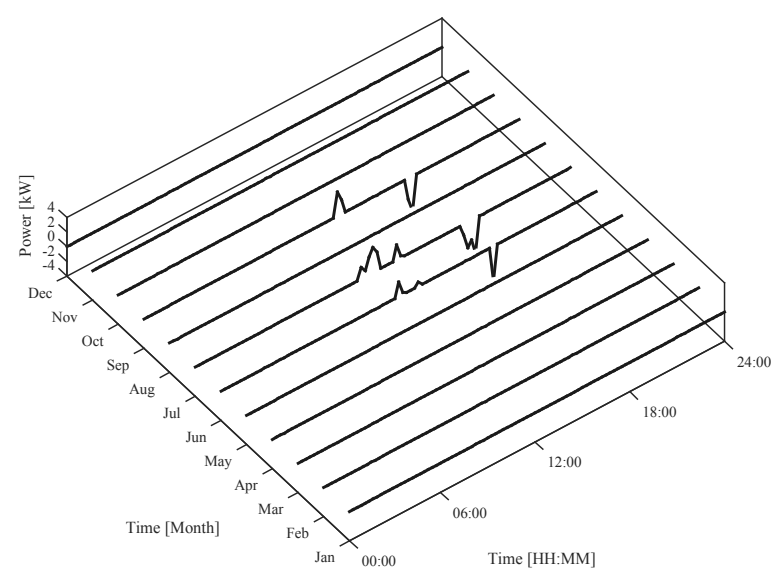

Fig. 7. Load profile of the integrated Energy Storage System.
Fig. 7 presents the load profile of the integrated ESS, where a positive/negative value is associated with a charging/discharging behaviour. The system operation in July, August and October is evident, with charging periods around noon and discharging at early evening when the LVG's demand exceeds the generation. During the remaining of the year, the ESS does not exchange energy with the LVG since the transformer's load limit is respected. To achieve the presented results a $2.8 \mathrm{kWh}$ battery would be necessary, with a charging peak power of $3.4 \mathrm{~kW}$. It is important to note that this battery capacity can be lower than the sum of 33 household batteries, considering the stochastic load behaviour. Although a coincident behaviour occurs, the stochasticity of the load implies small deviations on the individual peaks.

\section{CONCLUSIONS}

The study reported in this paper addresses the impacts introduced on distribution transformers' load due to the integration of NZEBs into existing LVGs. Moreover, this study analyses and proposes an Energy Storage System to mitigate transformer overloading due to reverse power flows.

To perform this evaluation, a LVG feeding 33 buildings is considered. The impacts introduced by integrating NZEBs into this LVG are assessed using several scenarios with distinct NZEB penetration levels. Then, assuming a worst case scenario in terms of transformer overloading, an Energy Storage System is integrated at transformer's output to mitigate the negative impacts.

Results show that for a reduced NZEB penetration level, transformer's yearly peak load decreases due to the local generation. However, for high penetration levels, the coincident PV generation, used by the buildings to achieve a net-zero energy status over a 1-year period, creates reverse power flows whose magnitudes achieve higher values than the evening demand peaks. For the worst case scenario, when all the 33 buildings are converted to NZEBs, the collected results reveal that the integrated Energy Storage System is effective in mitigating transformer overloading by storing the excessive generation to latter discharge when demand increases.

Since, in the case of NZEBs using PV generation, transformer overloading is found to occur mostly around noon, the integrated Energy Storage System could also be used to achieve distinct objectives during other periods of the day, instead of not being operated most of the time. For instance, local generation could be stored afternoon to reduce evening demand peak loads, even in days with no transformer overloading due to reverse power flows.

\section{ACKNOWLEDGMENT}

This work was supported by national funds through FCT - Fundação para a Ciência e a Tecnologia, under project UID/EEA/00066/2013 and European Union's Horizon 2020 research and innovation programme under the Storage4Grid project grant agreement No: 731155 . 


\section{REFERENCES}

[1] European Commission. Communication From The Commission To The European Parliament, The Council, The European Economic And Social Committee And The Committee Of The Regions - A Roadmap for moving to a competitive low carbon economy in 2050. 2011.

[2] S. Deng, R.Z. Wang, Y.J. Dai, How to evaluate performance of net zero energy building - A literature research, Energy. 71 (2014) 1-16.

[3] M. Santamouris, D. Kolokotsa, Passive cooling dissipation techniques for buildings and other structures: The state of the art, Energy and Buildings. 57 (2013) 74-94.

[4] E. Pyloudi, S. Papantoniou, D. Kolokotsa, Retrofitting an office building towards a net zero energy building, Advances in Building Energy Research. (2014) 1-14. doi:10.1080/17512549.2014.917985.

[5] European Union. Directive 2010/31/EU of the european parliament and of the council of 19 may 2010 on the energy performance of buildings (recast). Off J Eur Union 2010.

[6] PORDATA. Edifícios segundo os Censos: total e por época de $\begin{array}{llll}\text { construção } & \text { (in } & \text { Portuguese) }\end{array}$ http://www.pordata.pt/DB/Portugal/Ambiente+de+Consulta/Tabela (accessed July 14, 2017).

[7] M. Bollen, F. Hassan. Integration of Distributed Generation in the Power System. IEEE Press; 2011.

[8] Lopes RA, Magalhães P, Gouveia JoãPedro, Aelenei D, Lima C, Martins Joã, A case study on the impact of nearly Zero-Energy Buildings on distribution transformer aging, Energy (2018)

[9] Lopes RA, 2017, "Extending nearly Zero-Energy Buildings Load Matching Improvement to Community-Level", doctoral thesis, DEE FCT-NOVA, retrieved from http://hdl.handle.net/10362/29113.

[10] Pathomthat Chiradeja, R. Rmakumar "An approach to qualify the technical benefits of distributed generation" Energy Conversion, IEEE Transactions on, vol.19, no.4, pp.764,773, Dec. 2004.

[11] M. Thomson and D. G. Infield, "Impact of widespread photovoltaics generation on distribution systems," in IET Renewable Power Generation, vol. 1, no. 1, pp. 33-40, March 2007.

[12] S. Eftekharnejad, V. Vittal, G. T. Heydt, B. Keel and J. Loehr, "Impact of increased penetration of photovoltaic generation on power systems," in IEEE Transactions on Power Systems, vol. 28, no. 2, pp. 893-901, May 2013.

[13] V. F. Pires, O. Husev, D. Vinnikov and J. F. Martins, "A control strategy for a grid-connected PV system with unbalanced loads compensation," 2015 9th International Conference on Compatibility and Power Electronics (CPE), Costa da Caparica, 2015, pp. 154-159.

[14] B. Kroposki, DG Power Quality, Protection and Reliability Case Studies Report, Report No. NREL/SR-560-34635, National Renewable Energy Laboratory, Golden, CO, 2003.

[15] Rui Amaral Lopes, João Martins, Daniel Aelenei, Celson Pantoja Lima, A cooperative net zero energy community to improve load matching, Renewable Energy, Volume 93, 2016, Pages 1-13

[16] V. F. Pires, O. Husev, D. Vinnikov, and J. F. Martins, "A control strategy for a grid-connected pv system with unbalanced loads compensation," in 2015 9th International Conference on Compatibility and Power Electronics (CPE), June 2015, pp. 154-159.

[17] I. Roasto, A. Rosin, and T. Jalakas, "Power electronic interface converter for resource efficient buildings," in IECON 2017 - 43rd Annual Conference of the IEEE Industrial Electronics Society, 2017, pp. 3638-3643.

[18] I. Richardson, M. Thomson, D. Infield, C. Clifford, Domestic electricity use: A high-resolution energy demand model, Energy Build. 42 (10) (2010) 1878e1887.

[19] I. Richardson, M. Thomson, D. Infield, A high-resolution domestic building occupancy model for energy demand simulations, Energy Build. 40 (8) (2008) 1560e1566.

[20] Huld, T., Súri, M., Dunlop, E., Albuisson, M., Wald, L., 2005, Integration of HelioClim-1 database into PVGIS to estimate solar electricity potential in Africa. Proceedings from 20th European Photovoltaic Solar Energy Conference and Exhibition, 6-10 June 2005, Barcelona, SpainSdsd

[21] IEC, 2015, "60076-7 loading Guide for oil-immersed power transformers".

[22] Lopes RA, Magalhães P, Gouveia JoãPedro, Aelenei D, Lima C, Martins João, A case study on the impact of nearly Zero-Energy Buildings on distribution transformer aging, Energy (2018) 